\section{PWE-099 ANTIBIOTICS FOR SIBO: DO WE FIX IT?}

Catherine Sykes*, Robyn Cooke, Yan Yiannakou. County Durham and Darlington NHS Foundation Trust, Durham, UK

\subsection{6/gutjnl-2019-BSGAbstracts.419}

Introduction There is a lack of literature regarding symptomatology following diagnosis and treatment of small intestinal bacterial overgrowth $(\mathrm{SIBO})^{1}$. This service evaluation aimed to assess local outcomes.

Methods Retrospective assessment of patients referred for hydrogen $\left(\mathrm{H}_{2}\right)$ /methane $\left(\mathrm{CH}_{4}\right)$ breath tests $(\mathrm{BT})$ for SIBO was performed. From 201-017, 132 patients $(\mathrm{F}=94, \mathrm{M}=48)$ were tested at the University Hospital of North Durham. $50 \mathrm{~g}$ glucose in $250 \mathrm{ml}$ water was the substrate used for the tests. A positive BT was defined as an increase in hydrogen or methane of $\geq 10 \mathrm{ppm}$ with a starting baseline $<12 \mathrm{ppm}$ following a 12 hour fast and other accepted pre-test preparation. Main outcomes assessed were results from BTs, treatments given and outcomes following treatment. Quantitative statistics were not used due to small populations.

Results 159 BTs were conducted, with 28 reported as positive for SIBO. $79 \%$ of these patients were treated with relevant antibiotics (abx) (- week course). No major adverse effects of treatment were described. Following a positive BT, patients were reviewed after 4.5 months (range $0 .-2$ months). Subsequent follow up was 10 months (range -5 months) from initial test.

It is possible (though implausible) that all those returning for follow-up experienced symptom resolution. With this bold assumption, 23\% of patients (Outcome Category (OC) 1,2) may have experienced lasting improvement (Figure 1). We can be confident that some improvement was seen in $41 \%$ of the patients treated (OC 2,3,4). Disappointingly no patients were documented to have symptom improvement at second follow- up (OC 5). In 41\% of patients no improvement related to abx treatment was reported (OC 6,7).

Conclusions $\mathrm{Abx}$ can provide short term symptom relief in some patients with abdominal symptoms; however, this is generally not a long-term solution. There are important limitations of this work including the retrospective nature, variable follow-up duration and lack of validated symptom assessment. Findings do, however, support the need for improvements in understanding of maintaining a healthy microbiome. This maintenance is likely dependent on the aetiology of the underlying cause of the $\mathrm{SIBO}^{2}$.

\section{REFERENCES}

1. Shah SC, Day LW, Somsouk M \& Sewell JL. Meta-analysis: antibiotic therapy for small intestinal bacterial overgrowth. Aliment Pharmacol Ther. 2013; 38:92-34.

2. Rezaie A, Pimentel $M \&$ Rao $S$. How to test and treat small bowel bacterial overgrowth: an evidence-based approach. Curr Gastroenterol Rep. 2016; 18:8.

\section{Gastroenterology Service}

Orals

\section{OTH-01 A COMPLEX LOWER GASTROINTESTINAL POLYP MDT IMPROVES EVIDENCE-BASED DECISION MAKING AND EFFICIENCY OF ENDOSCOPY SCHEDULING}

Vinay Sehgal ${ }^{*}$, Alice Yearwood, Mehreen Chaudhry, Mark Samaan, Jonathan Fawkes, Marta Da Silva Teixeira, Melanie Corpus, Mr Charleston Mendoza, Edward Seward, Roser Vega. University College London Hospital, London, UK

\subsection{6/gutjnl-2019-BSGAbstracts.420}

Introduction Demand for lower gastrointestinal (LGI) endoscopy in the UK has doubled (1). In an increasingly ageing and co-morbid population, multi-disciplinary team (MDT)

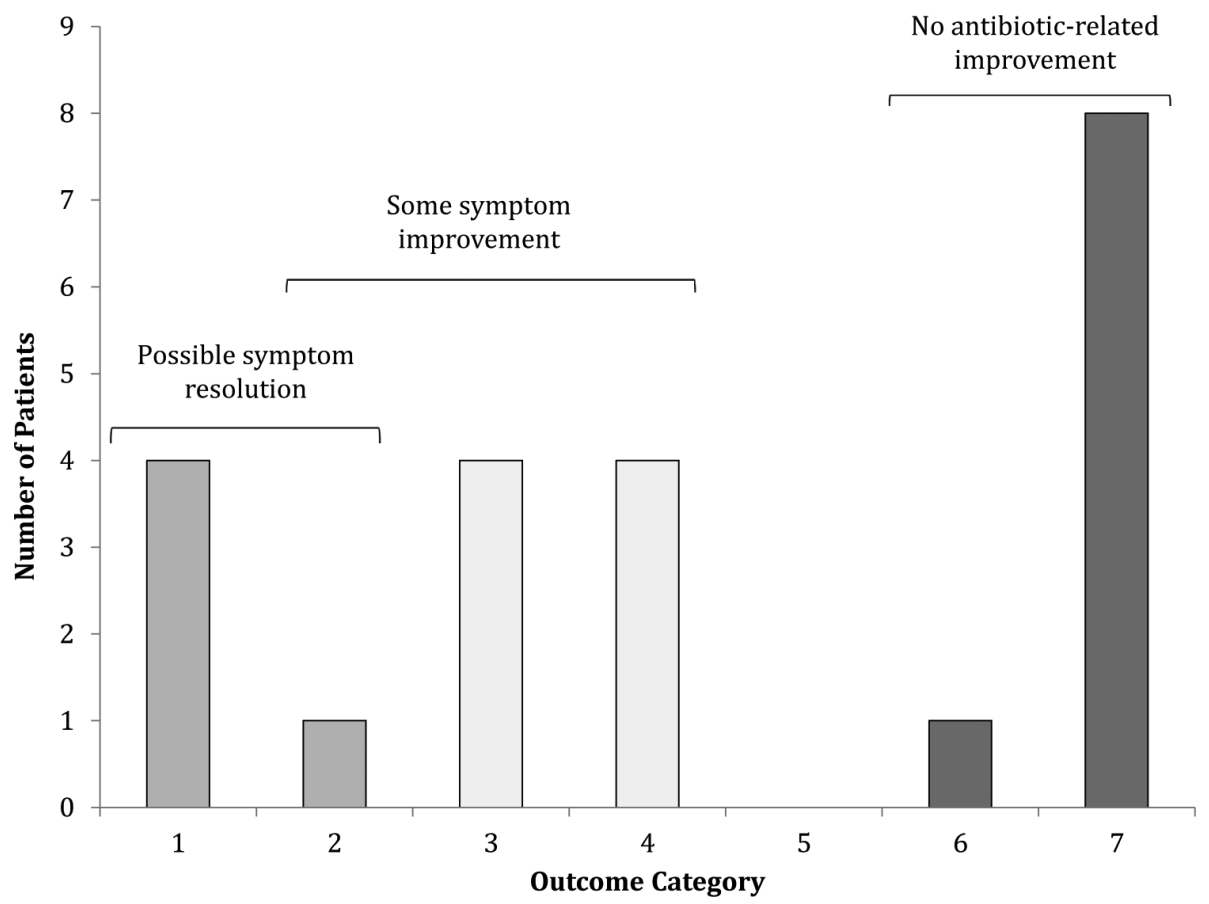

Abstract PWE099 Figure 1 Symptom outcomes following treatment for positive $\mathrm{H}_{2} / \mathrm{CH}_{4}$ BT. $1=$ =No follow-up; $2=$ Short term improvement with no further follow-up; $3=$ Short term improvement but symptom return by second appointment; $4=$ Improvement only while taking abx; $5=$ Stated symptom improvement at second follow-up; $6=$ Abx not taken but symptoms improved; $7=$ No improvement. 